\title{
Non-Autoimmune Hemolytic Anemia
}

National Cancer Institute

\section{Source}

National Cancer Institute. Non-Autoimmune Hemolytic Anemia. NCI Thesaurus. Code C34853.

Hemolytic anemia that is not mediated by immune mechanisms. 